\title{
Impact de boisements résineux sur la séquestration du carbone dans les Andes péruviennes : cas des plantations de Pinus patula dans la coopérative de Granja Porcon (Cajamarca), Pérou
}

Mathieu JONARD ${ }^{1}$

Renaud COLMANT ${ }^{1,2}$

Camille HeYLen ${ }^{1,2}$

Charles YSEBAERT ${ }^{1,}, 2$

Charles CARTON ${ }^{1}$

LUC PICARD ${ }^{1}$

Benoît CASSART ${ }^{1}$

Ablo Paul Igor Hounzandj ${ }^{1}$

Quentin PONETTE ${ }^{1}$

1 Université catholique de Louvain (UCL)

Earth \& Life Institute - Forest

sciences

Croix du Sud, 2 - L 7.05.09

1348 Louvain-la-Neuve

Belgique

2 Université catholique de Louvain (UCL)

Faculté des bioingénieurs

agronomique et environnementale (AGRO)

Croix du Sud, 2 - L7.05.01

1348 Louvain-la-Neuve

Belgique

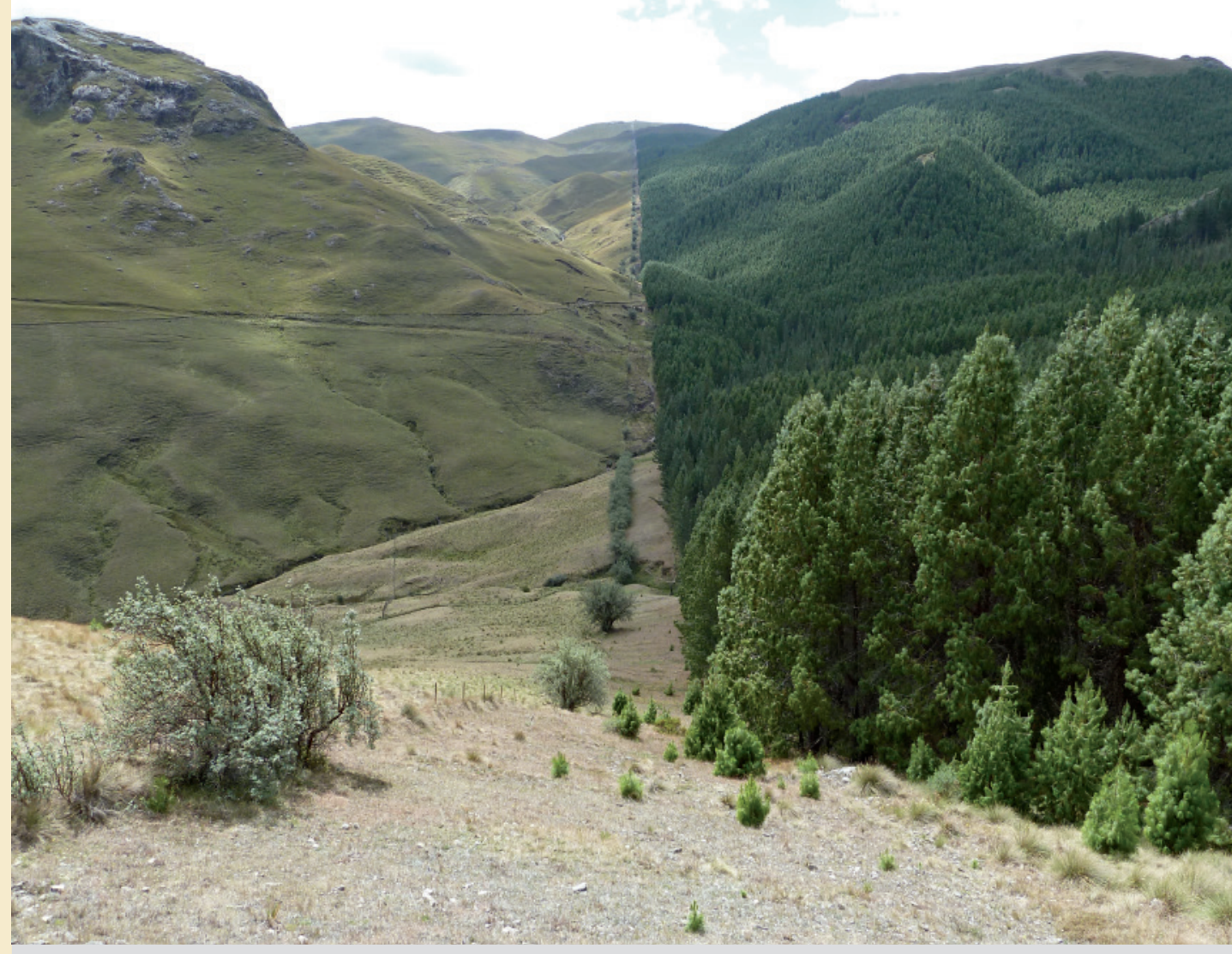

Photo 1.

Paysage d'altitude de la région de Cajamarca. La différence entre les deux occupations du sol rend compte des modifications induites par les reboisements. La coopérative de Granja Porcon se situe à droite de l'image, dans la partie boisée.

Photo C. Ysebaert, août 2011. 


\section{RÉSUMÉ}

\section{IMPACT DE BOISEMENTS RÉSINEUX SUR LA SÉQUESTRATION DU CARBONE DANS LES ANDES PÉRUVIENNES : CAS DES PLANTATIONS DE PINUS PATULA DANS LA COOPÉRATIVE DE GRANJA PORCON (CAJAMARCA)}

Dans la foulée de la réforme agraire de 1969, le gouvernement péruvien lança un programme de reboisement dans les Andes. Plus de 20000 ha furent ainsi boisés en pins dans la région de Cajamarca, dont 8500 ha dans la coopérative agraire de Granja Porcon. Destinés alors à approvisionner les paysans en bois et à développer l'activité économique locale, à enrayer la dégradation des sols et des forêts naturelles résiduelles, ces boisements sont aujourd'hui sollicités pour leur contribution au stockage du carbone. Cette recherche vise à comparer la capacité de séquestration de carbone des pâturages et des plantations de Pinus patula de haute altitude (3 250-3 $450 \mathrm{~m}$ ). À cette fin, deux approches ont été combinées : l'échantillonnage des sols (couches holorganiques et $0-100 \mathrm{~cm}$ ) et de la végétation dans les deux formations végétales (pâturages et boisements de Pinus patula âgés de 17 à 26 ans), sur cambisols développés sur matériaux volcaniques ; l'établissement d'un modèle de croissance de type peuplement, couvrant une plus grande diversité d'âges, de sylvicultures et de fertilités. Après vingt ans, la différence entre le carbone stocké dans les écosystèmes forestiers et les produits ligneux, d'une part, et en pâturages, d'autre part, s'élève à 154 t/ha, soit environ $7,7 \mathrm{t} / \mathrm{ha} / \mathrm{an}$. Cette différence s'explique très largement par la contribution des parties aériennes des arbres. Le reboisement en Pinus patula tend à réduire les stocks de carbone du sol par rapport aux formations prairiales correspondantes. La réduction est significative $(\alpha \leq 0,05)$ dans les 40 premiers centimètres du sol mais la dynamique temporelle suggère que cet effet est temporaire. Le modèle de croissance montre que le stockage de carbone dans les parties aériennes des arbres augmente avec l'âge et la densité des peuplements, ainsi qu'avec la fertilité du site. Il permet aussi d'évaluer la contribution des éclaircies à environ $23 \%$ de la quantité de carbone associée aux parties aériennes des arbres pour une sylviculture classique.

Mots-clés : Pinus patula, boisement, stockage de carbone, modèle de croissance, Andes péruviennes, Pérou.

\section{ABSTRACT}

\author{
IMPACT OF CONIFER PLANTATIONS \\ ON CARBON SEQUESTRATION IN THE \\ PERUVIAN ANDES: THE PINUS PATULA \\ PLANTATIONS IN THE GRANJA PORCON \\ COOPERATIVE (CAJAMARCA)
}

Following the 1969 agrarian reform, a reforestation programme in the Andes was launched by the Peruvian government. Over 20,000 ha of forests were planted in the Cajamarca region, including 8,500 ha in the Granja Porcon agrarian cooperative. At the time, the aims were to supply wood for peasant farmers, develop local economic activities and halt the degradation of soils and residual natural forests, but these forests are now being considered for their contribution to carbon sequestration. The aim of this study was to compare the carbon sequestration capacities of high-altitude (3,250-3,450 m) grasslands and Pinus patula plantations. Two approaches were used in combination: 1 ) soil sampling (holorganic layers and $0-100 \mathrm{~cm}$ ) and vegetation sampling in the two formations (grasslands and 17 to 26 year-old Pinus patula plantations), both on cambisols that developed from volcanic material; 2) development of a stand growth model covering a wider range of age classes, sylvicultural methods and degrees of soil fertility. After 20 years, carbon storage in forest ecosystems and woody material was higher by $154 \mathrm{t} / \mathrm{ha}$, or about $7.7 \mathrm{t} / \mathrm{ha}$ per year, than in grasslands. The difference is very largely accounted for by the contribution of the above-ground parts of the trees. Reforestation with Pinus patula tends to result in lower carbon storage in the forest soil than in the corresponding grassland formations. The difference is significant ( $\alpha$ $\leq 0.05)$ in the top $40 \mathrm{~cm}$ of the soil, but the dynamics over time suggest that this is a temporary effect. The growth model shows that carbon storage in the above-ground parts of the trees increases with the age and density of the stands, and with the fertility of the site. The model also assessed the contribution of thinnings at about $23 \%$ of the quantity of carbon associated with the above-ground parts of trees under conventional sylvicultural management.

Keywords: Pinus patula, plantation, carbon storage, growth model, Peruvian Andes, Peru.

\section{RESUMEN}

\author{
IMPACTO DE LA FORESTACIÓN CON \\ RESINOSAS EN EL SECUESTRO DE \\ CARBONO EN LOS ANDES PERUANOS: \\ CASO DE LAS PLANTACIONES DE PINUS \\ PATULA EN LA COOPERATIVA DE GRANJA \\ PORCÓN (CAJAMARCA)
}

Como complemento de la reforma agraria de 1969, el gobierno peruano impulsó un programa de reforestación en los Andes. De este modo, se repoblaron más de 20000 ha con pinos en el departamento de Cajamarca, 8500 de las cuales en la cooperativa agraria Granja Porcón. En un principio destinadas al suministro de madera y leña para los campesinos, al desarrollo de la actividad económica local y a frenar la degradación de suelos y bosques naturales residuales, actualmente también se utiliza el potencial de estas repoblaciones para contribuir al almacenamiento de carbono. Este estudio intenta comparar la capacidad de secuestro de carbono de pastos y plantaciones de Pinus patula en alta altitud (3 250-3 450 m). Para lograrlo, se combinaron dos enfoques: el muestreo de suelos (capas de mantillo forestal y 0-100 cm) y de la vegetación de las dos formaciones vegetales (pastos y bosques de Pinus patula de entre 17 y 26 años) en cambisoles formados sobre materiales volcáni$\cos y$, por otro lado, el establecimiento de un modelo de crecimiento de rodal que abarca una mayor diversidad de edades, cultivos silvícolas y fertilidades. Tras veinte años, los ecosistemas forestales y los productos madereros han almacenado $154 \mathrm{t} / \mathrm{ha}$ de carbono más que los pastos, lo que supone unas $7,7 \mathrm{t} / \mathrm{ha} / \mathrm{año}$. Esta diferencia se explica en gran medida por la contribución de las partes aéreas de los árboles. La reforestación con Pinus patula tiende a reducir las existencias de carbono en el suelo con respecto a las correspondientes formaciones de pastizales. La reducción es significativa ( $\alpha$ $\leq 0,05$ ) en los primeros $40 \mathrm{~cm}$ de suelo, pero la dinámica temporal sugiere que este efecto es transitorio. El modelo de crecimiento muestra que el almacenamiento de carbono en las partes aéreas aumenta con la edad y la densidad del rodal, así como con la fertilidad del sitio. Dicho modelo permite también evaluar la contribución de los raleos en aproximadamente un $23 \%$ de la cantidad de carbono asociada a las partes aéreas de los árboles con un manejo silvícola tradicional.

Palabras clave: Pinus patula, forestación, almacenamiento de carbono, modelo de crecimiento, Andes peruanos, Perú. 


\section{Introduction}

À la suite de la réforme agraire de 1969, le gouvernement péruvien soutenu par la coopération belge mit sur pied un vaste programme de développement de la région de Cajamarca (Pérou) dénommé Prodesca, dans lequel des boisements de plusieurs centaines d'hectares en eucalyptus puis en pins furent mis en œuvre avec des communautés paysannes.

Le besoin du Pérou en cellulose de pin conduisit à réaliser dans la coopérative agraire de Granja Porcon, située dans la zone du "Páramo ", un boisement industriel pilote de 3500 ha tout en veillant à améliorer les pratiques agropastorales, notamment par l'installation de terrasses et de réseaux d'irrigation prairiale.

Le succès de ce projet suscita l'intervention d'autres financements industriels qui portèrent l'aire boisée de Granja Porcon à 8500 ha constitués en majorité de pin patula, Pinus patula Schiede ex Schltdl. \& Cham., et de pin radiata, Pinus radiata $\mathrm{D}$. Don.

À l'origine, le boisement dans les Andes visait à pallier la carence en bois de feu, enrayer l'érosion des sols et la dégradation des forêts naturelles reliques, et développer l'activité économique locale. À Granja Porcon, toutefois, de nouvelles opportunités sont apparues au cours du temps. En effet, par leur impact profond sur les paysages, les forêts installées ont attiré de nombreux touristes péruviens et incité ainsi la coopérative à transformer ses productions primaires (lait, bois, laine, céréales) et à développer la vente de ses produits artisanaux. Dans le contexte de l'atténuation des changements climatiques, une nouvelle valorisation possible de ces boisements pourrait être liée à leur fonction de puits de carbone.

Dans le cadre du protocole de Kyoto signé en décembre 1997, des crédits carbone peuvent être échangés afin de permettre aux différents pays signataires de ce protocole de respecter leurs engagements. Ce marché du carbone repose, entre autres, sur le principe de la compensation des émissions de carbone dites « non évitables » en payant une autre entité pour qu'elle séquestre des gaz à effet de serre. Ainsi, par le biais du Mécanisme pour un développement propre (MDP) mis en place dans le cadre du protocole de Kyoto, une entreprise d'un pays industrialisé peut financer un projet de réduction des émissions de $\mathrm{CO}_{2}$ ou de séquestration du carbone tel que le boisement dans un pays en développement et bénéficier de crédits carbone. L'obtention de crédits carbone pourrait être un moyen de financer de nouveaux boisements et de dynamiser la gestion des plantations existantes.

Afin de pouvoir bénéficier de crédits carbone, il faut pouvoir démontrer que le boisement contribue globalement à une séquestration accrue de carbone et quantifier cette augmentation. Dans ce contexte, l'objectif de l'étude est double : quantifier l'évolution des stocks de carbone dans deux compartiments de l'écosystème (sol et partie aérienne des arbres) suite au boisement de pâturages extensifs dans le domaine de Granja Porcon ; caractériser la dynamique de croissance du pin patula en fonction de la station (altitude, type de sols) et de la sylviculture pratiquée afin de fournir aux forestiers andins un outil d'aide à la gestion des plantations (planification des coupes, estimation des potentialités de production). Le modèle de croissance établi pourra être utilisé dans le cadre de la quantification des stocks de carbone pour évaluer les quantités de carbone associées aux arbres prélevés en éclaircie et pour prédire l'évolution des stocks aériens de carbone à l'échelle de la coopérative et de la région.

\section{Matériels et méthodes}

\section{Zone d'étude}

La coopérative Atahualpa-Jerusalén de Granja Porcon se situe dans le département de Cajamarca au Nord-Ouest du Pérou, entre les coordonnées géographiques $06^{\circ} 56^{\prime}$ ' et $07^{\circ} 05^{\prime}$ de latitude Sud et $78^{\circ} 32^{\prime}$ et $78^{\circ} 43^{\prime}$ de longitude Ouest. L'altitude varie entre 3000 et $4000 \mathrm{~m}$ et le terrain, accidenté, peut présenter des pentes fortes atteignant $75 \%$ (Kometter et Palomares, 1994). Le climat est de type tempéré. Les précipitations moyennes annuelles sont comprises entre $750 \mathrm{~mm}$ et $1200 \mathrm{~mm}$ et la température moyenne annuelle est de $10^{\circ} \mathrm{C}$ (Ministerio de agricultura, 1979). Les sols sont majoritairement issus de roches volcaniques (61\%) et de grès

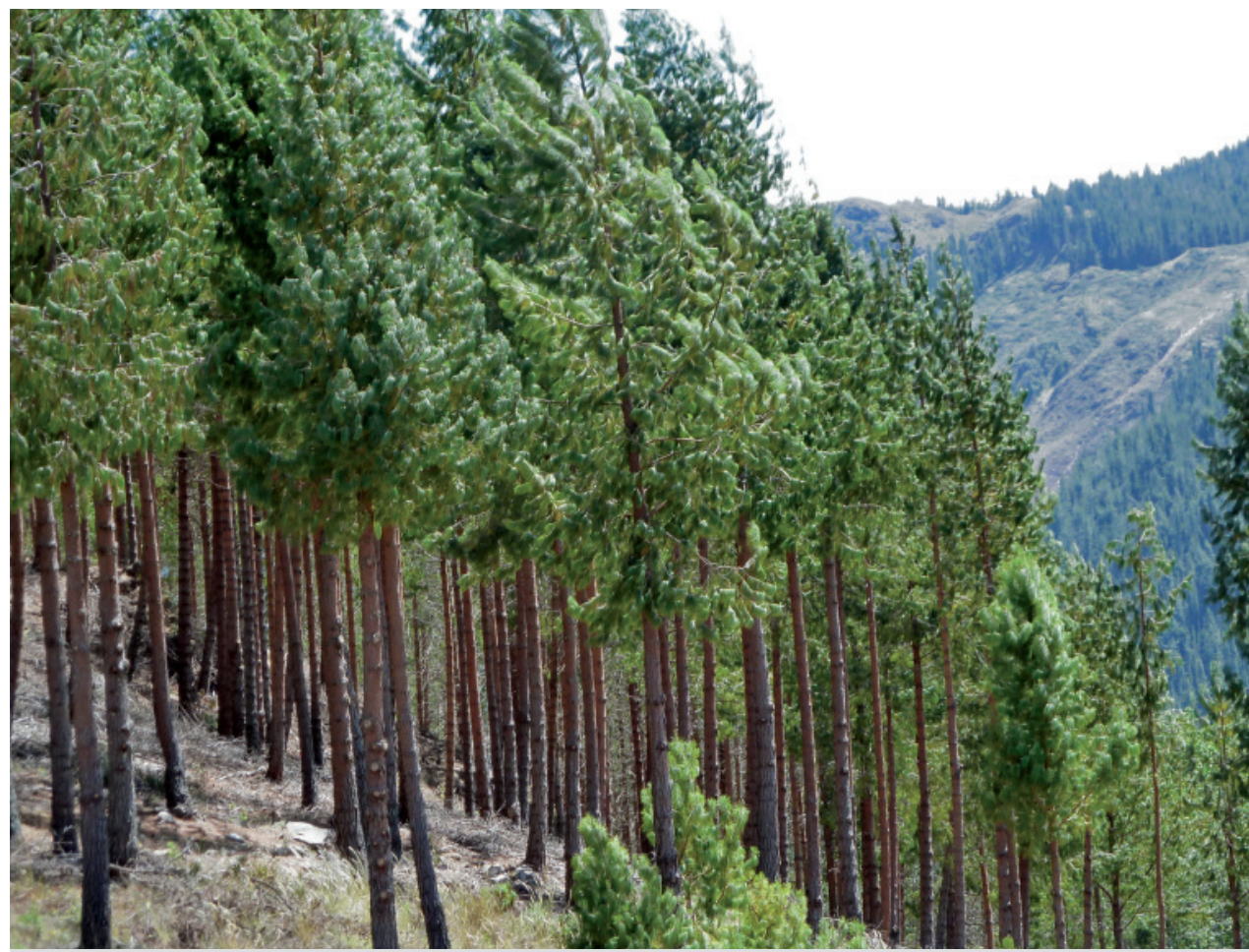

Photo 2.

Boisement de Pinus patula âgé de 17 ans et situé à une altitude de $3200 \mathrm{~m}$, dans la coopérative de Granja Porcon. Tous les arbres ont été élagués. Photo C. Ysebaert, août 2011. 
(35\%) (Ministerio de agricultura, 1979), et présentent des teneurs élevées en matière organique. En dehors des vallons et des zones boisées, la formation végétale dominante est le " Páramo », caractérisée par des graminées comme Stipa sp., Calamagrostis sp., Festuca sp., etc. La végétation arborescente et arbustive naturelle se localise préférentiellement aux abords des cours d'eau (par exemple, Alnus acuminata, Polylepis racemosa, Gynoxis sp.). Les plantations occupent actuellement plus de 7500 ha et sont constituées principalement de Pinus patula et Pinus radiata (Adefor, 2005).

\section{Dynamique de croissance des peuplements}

La modélisation de la dynamique de croissance des peuplements de pin patula a été réalisée sur la base de données collectées dans 20 placettes permanentes de $0,5(n=18)$ ou 1 ha $(n=2)$ et 48 placettes temporaires de 7 ares. Les placettes permanentes ont été installées au début des années 1990 et ont fait l'objet de mesures annuelles de 1993 à 2000 (diamètre à 1,3 m de hauteur, et hauteur totale de chaque arbre à l'aide d'une perche graduée) ; 14 d'entre elles ont été remesurées en 2011 en utilisant cette fois un dendromètre vertex pour la mesure de la hauteur totale. La gamme d'âge balayée va de 5 à 28 ans avec une absence de données entre 16 et 24 ans. Afin de compléter ce premier jeu de données, des mesures (diamètre à 1,3 m de hauteur des arbres, et hauteur dominante) ont été effectuées en 2011 dans 48 placettes temporaires choisies afin de couvrir de manière homogène les différentes classes d'âge, d'altitude et de profondeur de sol. Pour les deux types de placettes, plusieurs facteurs du milieu ont été relevés : la pente, la position dans le versant (bas de versant / fond de vallée, milieu de versant, sommet de versant / plateau), le matériau parental (volcanique, gréseux, gréseux-volcanique), la profondeur de sol, l'altitude (gamme couverte : 3000 à 3630 m) et l'exposition. Le modèle de croissance (tables de production) a été élaboré sur la base de quatre relations dendrométriques fondamentales (Skovsgaard et Vanclay, 2008). La première relation décrit l'évolution de la hauteur dominante en fonction de l'âge pour différentes classes de fertilité (courbes de productivité). Elle est basée sur la première loi d’Eichhorn qui stipule que la hauteur dominante d'un peuplement équienne monospécifique n'est fonction que de l'âge, de l'essence, et des conditions de la station (Eichhorn, 1904). Plusieurs types de modèles mathématiques ont été testés et celui s'ajustant le mieux aux données a été retenu. La deuxième relation a permis de prédire la production totale en volume sur la base d'un indice de productivité (hauteur dominante à un âge donné), de l'âge et de la surface terrière à un âge donné (Clutter, 1963). La troisième relation découle de l'hypothèse de réponse à l'éclaircie (Wiedemann, 1951) qui suppose que l'éclaircie n'influence pas significativement la production totale en volume dans une large gamme d'intensité ( $<5 \%$ en surface terrière). La dernière relation correspond à la loi d'auto-éclaircie qui décrit l'évolution du nombre de tiges en fonction du diamètre moyen dans un peuplement non éclairci. Trois types de sylviculture ont été considérés : une sylviculture sans éclaircie, une sylviculture traditionnelle (éclaircies tous les six ans) et une sylviculture plus dynamique (éclaircies tous les trois ans). Selon la classe de fertilité et le type de sylviculture, l'intensité des éclaircies a été déterminée en veillant à produire des accroissements plus ou moins constants, à maintenir sur pied une densité supérieure au seuil d'apparition de la concurrence $\left(20 \mathrm{~m}^{2} / \mathrm{ha}\right.$ à 15 ans ; Jonard, 1999) et à ne pas prélever plus de $50 \%$ de la surface terrière. Pour la modalité sans éclaircie, l'évolution du nombre de tiges a été déduite de la droite d'auto-éclaircie.

\section{Stockage du carbone}

Les stocks de carbone ont été quantifiés pour quatre formations végétales, correspondant à deux occupations du sol contrastées : des pâturages de deux types (sur brûlis et simples); des boisements de Pinus patula de deux classes d'âge (17 ans et 25 ans). Pour chaque formation végétale, trois placettes carrées de 4 ares ont été délimitées dans deux zones de conditions écoclimatiques comparables (topographie, type de sol). Les sites retenus pour cette partie de l'étude se localisent entre 3250 et 3450 m d'altitude, sur des versants exposés au Nord ou Nord-Est ; ils sont situés sur des cambisols ou des regosols (WRB ${ }^{1}$; Driessen et al., 2001) riches en matière organique, développés pour la plupart sur des roches volcaniques.

\section{Stockage du carbone dans les sols et la végétation herbacée}

Dans chaque placette, la végétation herbacée (parties aériennes), les horizons holorganiques et les couches minérales de sol (0-10, 10-20, 20-40, 40-60, 60-80, 80-100 cm) ont été prélevés en neuf points, puis rassemblés pour constituer un échantillon composite par placette (et profondeur). La densité apparente de la terre fine a été estimée en prélevant un cylindre de $100 \mathrm{~cm}^{3}$ par placette et profondeur. Après détermination des poids secs par unité de surface, les échantillons organiques (végétation herbacée, horizons holorganiques) ont été broyés en vue du dosage du carbone (C) et de l'azote $(\mathrm{N})$ par combustion sèche. Pour les couches minérales, le $\mathrm{pH}\left(\mathrm{H}_{2} \mathrm{O}, \mathrm{CaCl}_{2}\right)$ et les teneurs totales en $\mathrm{C}$ et $\mathrm{N}$ (combustion sèche) ont été mesurés après tamisage à $2 \mathrm{~mm}$ sur tous les échantillons de sols (Heylen, 2012).

Les effets "formation végétale » (4 modalités : pâturages simples / sur brûlis, boisements de Pinus patula de 17 ans / 25 ans) ou « occupation du sol » (2 modalités : pâturages / boisements) ont été testés indépendamment à l'aide d'une Anova 1, prenant en compte l'effet aléatoire « site ». En cas d'effet « formation végétale » significatif $(\alpha \leq 0,05)$, un test de Tukey a été réalisé.

\section{Stockage du carbone dans les parties aériennes des arbres}

Au niveau des plantations, la circonférence à 1,3 m de hauteur (C130) et la hauteur totale (Htot) ont été mesurées sur l'ensemble des arbres de chaque placette. Les caractéristiques générales des peuplements échantillonnés sont reprises dans le tableau I.

Sur la base des inventaires dendrométriques, deux arbres ont été abattus dans chaque placette, en veillant à couvrir la gamme de variation des circonférences de chaque peuplement. Après détermination de la longueur totale du tronc, la circonférence a été mesurée à 1,3 m ainsi qu'à

${ }^{1}$ WRB : World Reference Base for Soil Resources (WRB). 
Tableau I.

Principales caractéristiques dendrométriques des peuplements.

\begin{tabular}{|c|c|c|c|c|c|}
\hline Peuplement & Âge (ans) & Densité (N/ha) & $G^{1}\left(m^{2} / h a\right)$ & $\operatorname{Hdom}^{2}(\mathrm{~m})$ & $\mathrm{C} 130^{3}(\mathrm{~cm})$ \\
\hline 1 & 17 & 1292 & 60,3 & 20,0 & $74,8 \pm 16,7$ \\
\hline 2 & 17 & 1308 & 55,9 & 19,1 & $70,6 \pm 19,7$ \\
\hline 3 & 25 & 483 & 45,3 & 25,8 & $104,8 \pm 28,3$ \\
\hline 4 & 26 & 792 & 71,3 & 24,3 & $103,3 \pm 25,7$ \\
\hline
\end{tabular}

2,5 m de hauteur, puis tous les 2,5 m jusqu'à la découpe $22 \mathrm{~cm}$. La longueur du fin bout, de même que sa circonférence à mi-longueur, ont également été mesurées. Un inventaire complet des branches a ensuite été réalisé en relevant le diamètre des branches à $10 \mathrm{~cm}$ de l'insertion sur le tronc (D10) et la distance à l'apex.

Le tronc a été découpé en billons ; chaque billon ainsi que le fin bout ont été pesés sur le terrain. Des disques de $2 \mathrm{~cm}$ d'épaisseur ont alors été prélevés à la base de chacun d'eux ainsi qu'au milieu du fin bout pour détermination des teneurs en eau $\left(65^{\circ} \mathrm{C}\right)$ et en $\mathrm{C}$. Trois branches balayant la gamme de diamètres rencontrés ont également été prélevées sur chaque arbre abattu pour détermination des teneurs en eau $\left(65^{\circ} \mathrm{C}\right)$ et en $\mathrm{C}$ des aiguilles et des parties ligneuses (les parties ligneuses des branches sont appelées «branches » dans la suite du document).

Une première série de modèles ont été établis pour estimer la masse sèche des aiguilles ou du bois à l'échelle de la branche, à partir du diamètre des branches (D10). Ces modèles ont permis d'estimer la biomasse de ces mêmes compartiments à l'échelle du houppier des arbres abattus, en s'appuyant sur l'inventaire des branches. Dans une seconde étape, la biomasse du tronc (bois + écorce), des aiguilles et des parties ligneuses (bois + écorce) des branches a été estimée à l'échelle de l'arbre en ajustant l'équation allométrique (équation 1). Selon Genet et al. (2011), cette équation donne de bons résultats pour tous les compartiments de l'arbre.

$Y=a+b \times\left(C 130^{2} . \text { Htot }\right)^{\mathrm{Y}}+\varepsilon$

(équation 1)

Avec :

- $Y$, biomasse du compartiment considéré (kg) ;

- C130, circonférence du tronc à 1,3 m de hauteur (m) ;

- Htot, hauteur totale de l'arbre (m) ;

- $a, b$ et $\mathrm{\gamma}$, paramètres à estimer ;

- $\varepsilon$, terme d'erreur représentant la variabilité non expliquée du modèle.

De façon à prendre en compte l'hétéroscédasticité, la variance des résidus a été modélisée à l'aide d'une fonction puissance. Le modèle a été décliné en quatre formes de base différentes en considérant les cas particuliers où $a=0$ et/ou $\gamma=1$. Les ajustements ont été effectués par la méthode du maximum de vraisemblance en testant pour un effet âge (17 vs 25 ans) éventuel sur le(s) paramètre(s). Le choix du meilleur modèle s'est basé sur les critères AIC, BIC et sur le test du rapport de vraisemblance. Les performances finales du modèle retenu ont été caractérisées par le $R^{2}$ et l'écart-type résiduel $\left(S_{Y}\right)$ (Rondeux, 1999).

La quantité de carbone a été calculée en multipliant la biomasse du compartiment considéré par la concentration moyenne en carbone correspondante. La biomasse totale (le stock de carbone total) à l'hectare a été obtenue en sommant les estimations de chacun des compartiments pour l'ensemble des arbres de la placette et en divisant ensuite par la surface de la placette.

\section{Résultats}

\section{Dynamique de croissance des peuplements}

L'analyse et le traitement des données de hauteur dominante en fonction de l'âge des peuplements nous ont permis d'établir cinq courbes de productivité délimitant quatre classes de fertilité : $A, B, C$ et $D$ (figure 1 ). Le modèle établi utilise l'équation de Chapman-Richards et la méthode de régression sériée. L'allure des différentes courbes de productivité dépend de l'indice de productivité qui correspond à une hauteur dominante à un âge de référence fixé à 12,5 ans. La figure 1 montre que la distribution des observations concorde bien avec les classes de fertilité établies.

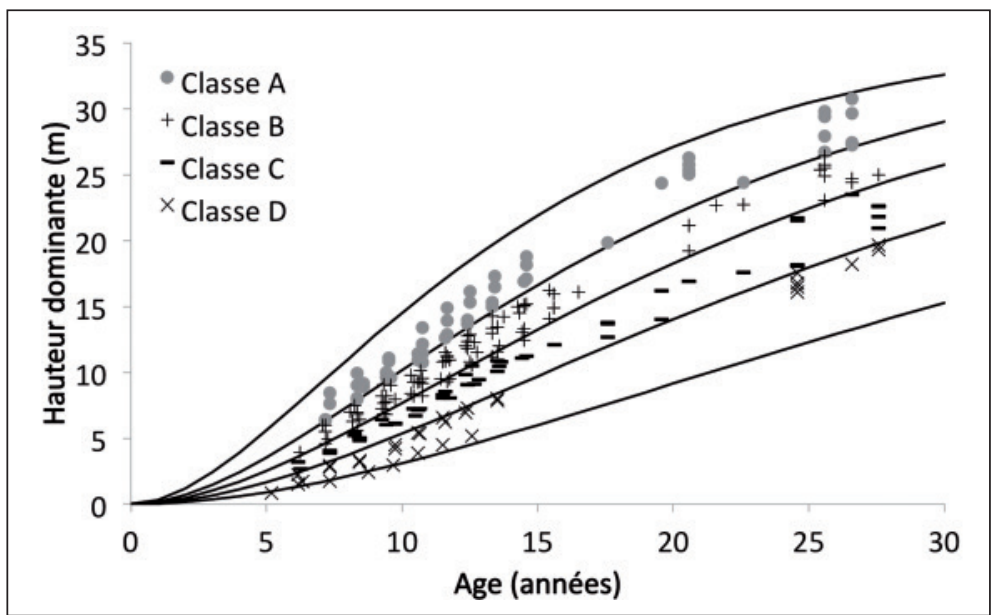

Figure 1

Courbes de productivité pour Pinus patula dans les Andes péruviennes (domaine de Granja Porcon), établies en considérant des hauteurs dominantes de $4,5,7,5,10,5,13,5,18,5 \mathrm{~m}$ à 12,5 ans. 


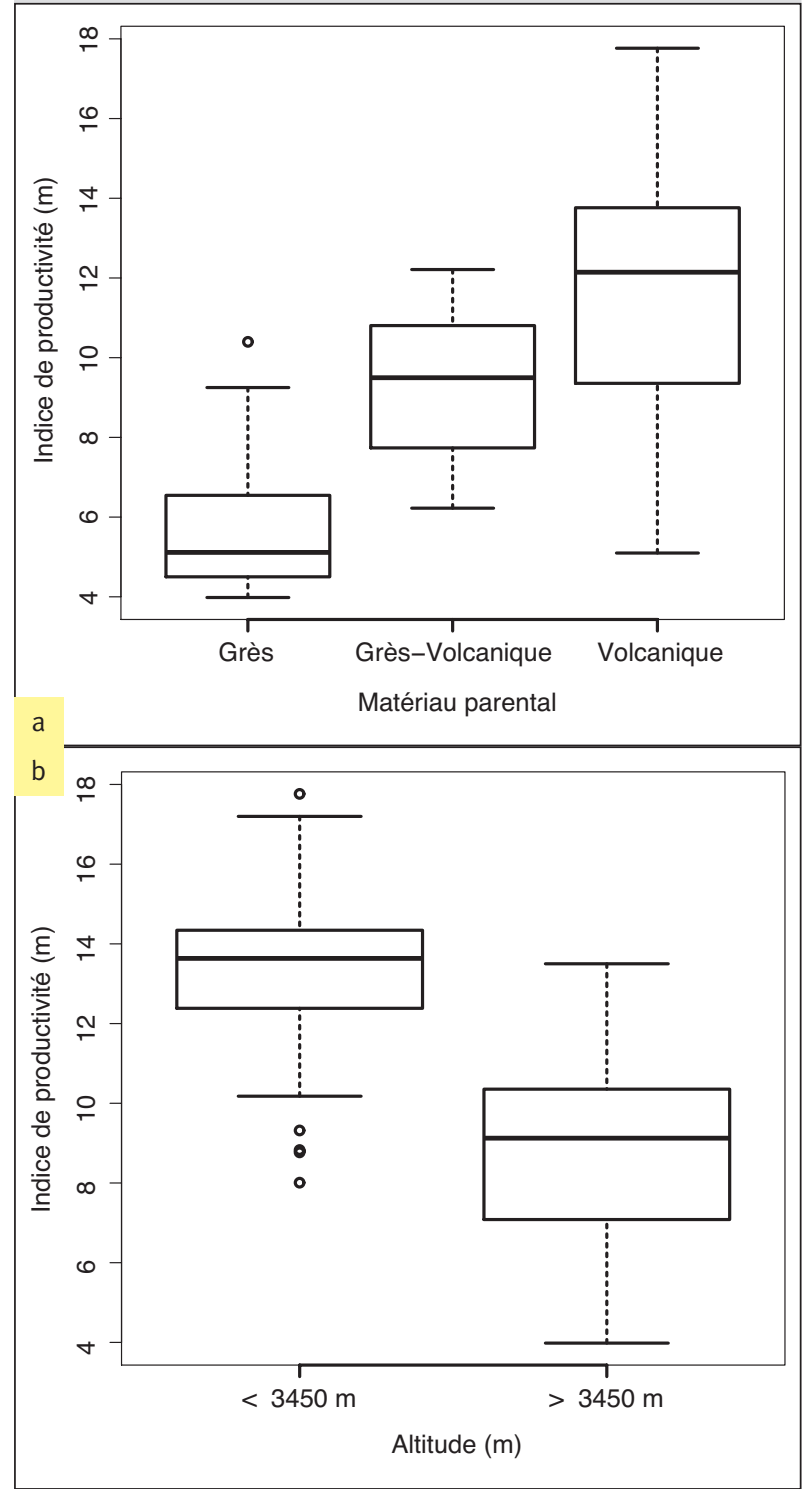

Figure 2.

Impact du matériau parental (a) et de l'altitude (b) sur l'indice de productivité (hauteur dominante à 12,5 ans) pour Pinus patula dans les Andes péruviennes (Granja Porcon). Pour chaque facteur, les modalités sont significativement différentes les unes des autres $(\alpha=0,05)$.

Les six facteurs du milieu relevés dans les deux types de placettes d'échantillonnage $(n=68)$ ont été mis en relation avec l'indice de productivité. Parmi ces six facteurs, deux seulement ont un effet significatif sur la productivité : le matériau parental et l'altitude. Ensemble, ces deux facteurs expliquent $60 \%$ de la variabilité de l'indice de productivité. Comme indiqué dans la figure 2, la productivité des peuplements de pin patula est plus élevée pour les sols ayant un matériau parental en partie ou entièrement constitué de roche volcanique par comparaison à ceux constitués de grès. Cette productivité a tendance à être plus élevée pour des peuplements situés en dessous de 3450 m d'altitude.

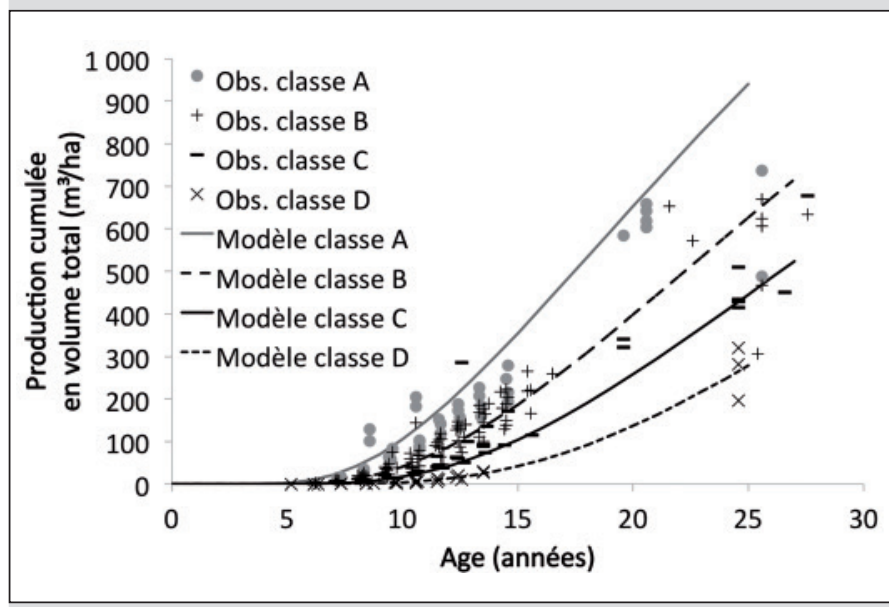

Figure 3.

Évolution temporelle de la production totale en volume en fonction de l'âge et de la classe de fertilité pour Pinus patula dans les Andes péruviennes (domaine de Granja Porcon).

L'évolution temporelle de la production totale en volume a été estimée sur la base du modèle de Clutter (1963). Dans la figure 3, des courbes moyennes issues de ce modèle ont été représentées par classe de fertilité afin de pouvoir les confronter aux observations.

Afin d'illustrer les tables de production, l'évolution du diamètre moyen et du nombre de tiges à l'hectare a été mise en relation avec la hauteur dominante pour la classe de fertilité $A$ et les trois types de sylviculture (figure 4). Le nombre de tiges commence à diminuer vers une hauteur dominante de 16 et $15 \mathrm{~m}$, respectivement, pour les sylvicultures traditionnelle ou dynamique, alors qu'il faut attendre $23 \mathrm{~m}$ de hauteur dominante pour observer une diminution de la densité dans la modalité sans éclaircie. L'évolution de la densité est assez semblable pour les sylvicultures traditionnelles et dynamiques, si ce n'est que la diminution du nombre de tiges dans la sylviculture dynamique se produit plus fréquemment mais avec une intensité moindre à chaque intervention. Cette évolution de la densité pour les sylvicultures traditionnelles et dynamiques permet de maintenir un accroissement constant du diamètre moyen alors que celui-ci diminue en l'absence d'éclaircie.

\section{Stockage du carbone dans les sols}

En considérant les couches holorganiques et les 100 premiers centimètres du sol minéral, le stock de carbone (moyenne \pm écart-type) varie respectivement entre 20,1 \pm 6,4 et $34,1 \pm 9,9 \mathrm{~kg} / \mathrm{m}^{2}$ dans les peuplements de 16 ans et dans les pâturages parcourus par les feux; il est comparable dans les deux autres formations, où il totalise respectivement $29,0 \pm 8,4$ et $28,4 \pm 7,5 \mathrm{~kg} / \mathrm{m}^{2}$ pour les pâturages simples et les boisements de 25 ans. La différence entre formations végétales est très proche du niveau de signification $(P=0,0521)$; il n'y a pas de différence significative $(P=0,1109)$ entre occupations du sol (pâturage versus plantation). En considérant chaque couche séparément, les stocks de carbone s'avèrent significativement supérieurs 


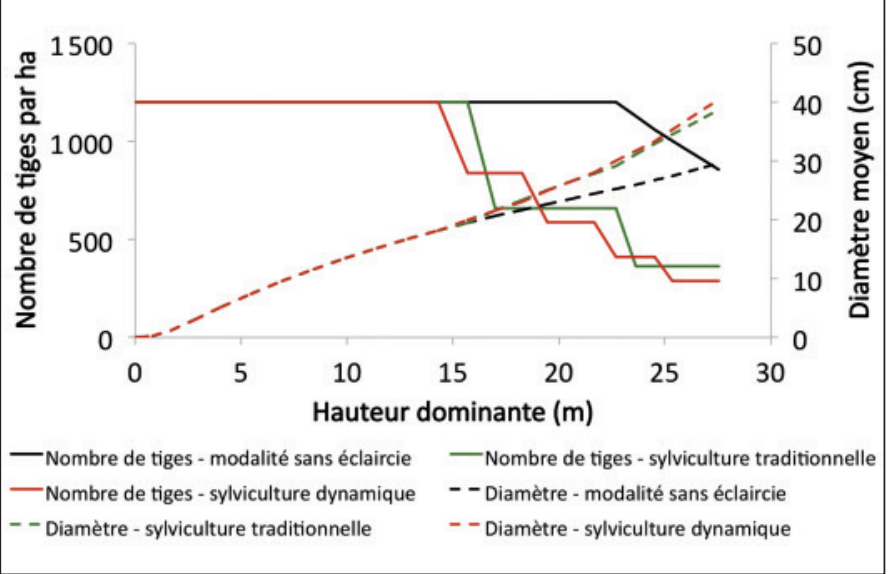

Figure 4.

Évolution du diamètre moyen et du nombre de tiges par hectare en fonction de la hauteur dominante pour la classe de fertilité $A$ et trois types de sylviculture différents.

sous pâturages dans les couches $0-10 \mathrm{~cm}(P=0,0056), 10$ $20 \mathrm{~cm}(P=0,0183)$ et $20-40 \mathrm{~cm}(P=0,0476)$; au-delà de cette profondeur, les stocks de carbone ne diffèrent pas significativement selon la formation végétale ou l'occupation du sol (figure 5).

Au total, la contribution des couches holorganiques et des 40 premiers centimètres du sol minéral varie entre $60 \%$ (reboisements de 25 ans) et $70 \%$ (autres formations) du stock total de carbone des sols. Les stocks moyens par horizon dans les 40 premiers centimètres peuvent atteindre 80 tonnes de carbone à l'hectare (figure 5). La différence de distribution du carbone sous pâturages et plantations s'ex- plique par : les racines, plus fines, plus rapidement décomposables, et plus importantes sous pâturages (Stevenson et Cole, 1999) ; les restitutions de matière organique, différentes en termes de quantité et de composition (rapport carbone/azote plus élevé sous plantations) (Laganière et al., 2010) ; la bioturbation réalisée par des espèces plus exploratrices (espèces endogées) sous pâturages que sous forêts (Tonneijck et Jongmans, 2008).

Par ailleurs, comme le suggère la figure 6 , le reboisement semble d'abord s'accompagner d'une réduction du stock de carbone dans les sols jusqu'à l'obtention d'une valeur minimale, puis d'une réaugmentation. En première approche, cette évolution peut être représentée par une équation polynomiale du second degré :

$$
\begin{aligned}
& Y=0,0659 \times a g e^{2}-1,7974 \times \text { age }+29,9842\left(R^{2}=0,31\right) \\
& \text { (équation 2) }
\end{aligned}
$$

Avec :

- $Y$, stock de carbone organique dans les couches minérales du sol 0-100 cm $\left(\mathrm{kg} \mathrm{C} / \mathrm{m}^{2}\right)$;

- age, temps depuis la plantation (ans).

Selon cette équation, la valeur minimum serait obtenue la treizième année et s'élèverait à environ 60 \% du stock de carbone initial. Ensuite, le stock de carbone du sol se reconstituerait progressivement pour atteindre le niveau de départ après une trentaine d'années. Si cette évolution est en accord avec de nombreux résultats de la littérature (notamment Turner et Lambert, 2000 ; Guo et Gifford, 2002 ; Guo et al., 2007 ; Laganière et al., 2010), elle doit néanmoins être confirmée par un échantillonnage balayant une gamme plus large d'âges de peuplements et de stations.

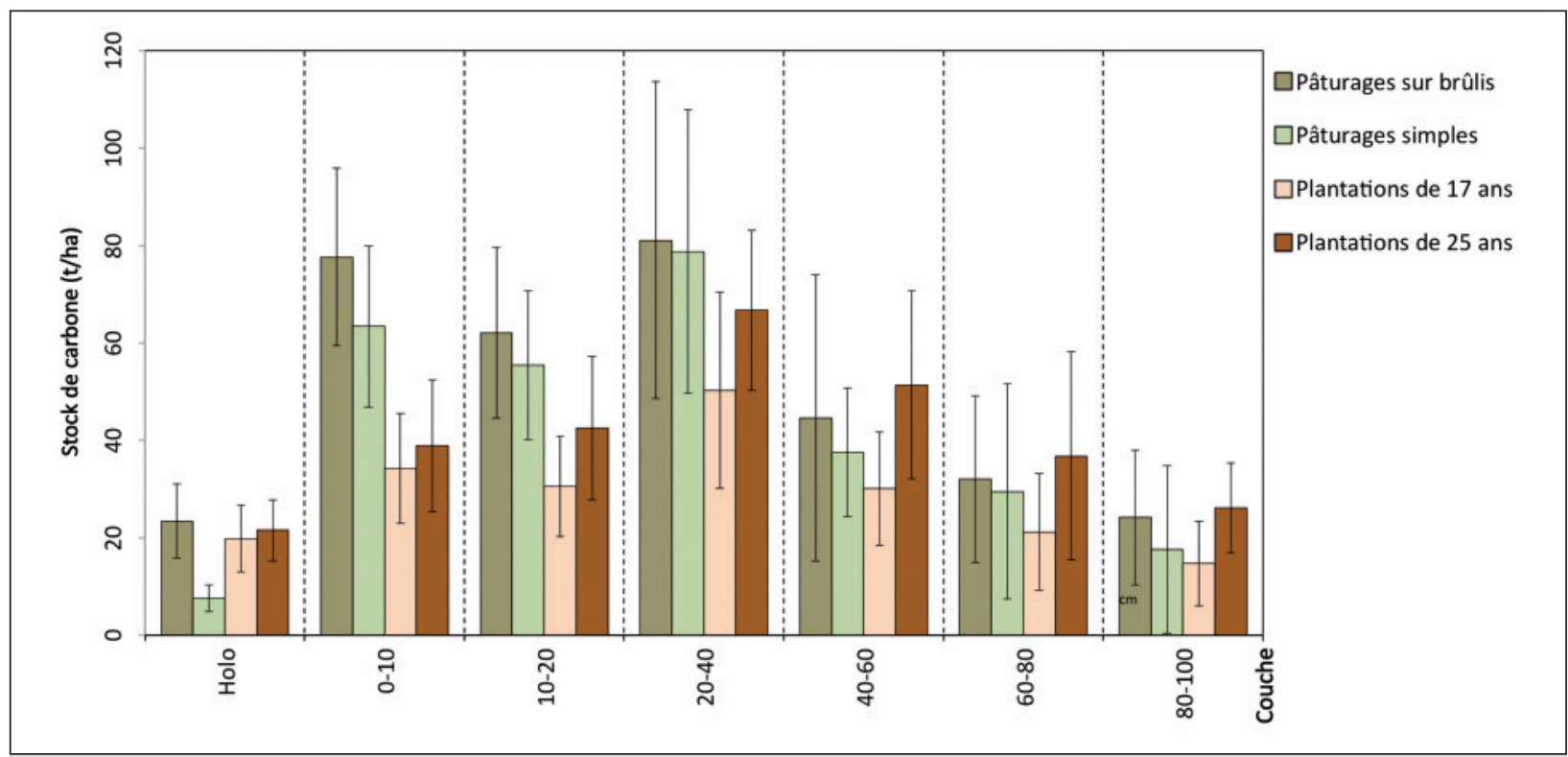

Figure 5.

Évolution du stock de carbone organique selon la profondeur $(\mathrm{cm})$ et la formation végétale (moyennes \pm écarts-types). 


\title{
Discussion et conclusions
}

\section{Dynamique de croissance des peuplements}

Une différence sensible de productivité existe entre les classes de fertilité présentes dans la zone d'étude. Chacune de ces classes peut subir une sylviculture particulière selon sa productivité propre. En effet, des interventions plus dynamiques sont préconisées pour les classes de fertilité élevées. Dans le cas présent, la sylviculture dynamique diffère principalement de la sylviculture pratiquée par l'âge plus précoce auquel intervient la première éclaircie et par les rotations plus courtes (trois ans au lieu de six). L'intensité globale en termes de nombre de tiges enlevées

Figure 6.

Évolution temporelle du stock de carbone, d'une part, dans les couches holorganiques et minérales (en gris), et dans les couches minérales, d'autre part (en noir). Moyennes en traits pleins, intervalle de confiance à $95 \%$ en pointillé.

\section{Stockage du carbone dans les parties aériennes des arbres}

Pour les trois compartiments considérés, les ajustements ont permis de retenir une forme simplifiée de l'équation 1 avec $a=0$ et $\gamma=1$ (tableau II). À titre d'exemple, la relation obtenue pour la biomasse du tronc $\left(B_{\text {tronc }}\right)$ est illustrée à la figure 7.

Comme démontré par Genet et al. (2011), le paramètre $b$ peut être interprété comme le produit d'un coefficient de forme ajusté et de la masse volumique. En intégrant la hauteur totale, cette équation permet de prendre en compte la différence de biomasse existant entre deux arbres de même circonférence à 1,3 m mais de hauteur différente. Pour les quatre peuplements étudiés, les équations allométriques ont permis d'estimer, à l'échelle de l'arbre, l'évolution de la biomasse totale et de la contribution des trois compartiments en fonction de la classe de grosseur (figure 8); dans le cas présent, la hauteur totale a été estimée à partir d'une relation liant cette dernière à la circonférence à 1,3 m. Comme attendu, on observe que la contribution du tronc à la biomasse aérienne totale augmente avec la taille de l'arbre. Pour l'arbre moyen des peuplements étudiés (C130 $\approx 90 \mathrm{~cm}$; tableau I), la biomasse aérienne totale s'élève à près de $290 \mathrm{~kg}$, pour une contribution des compartiments tronc, branches et aiguilles d'environ $70 \%, 24 \%$ et $6 \%$, respectivement (figure 8 ). reste relativement proche, ce qui limite le gain d'accroissement en diamètre. En revanche, la progressivité des interventions permet d'obtenir des accroissements radiaux plus réguliers, tout en limitant les risques d'instabilité des peuplements et d'érosion des sols.

\section{Capacité de séquestration du carbone par les plantations de Pinus patula}

L'impact du reboisement sur un cycle sylvicole d'une vingtaine d'années peut être discuté au regard de la figure 9. Pour chaque occupation du sol, les stocks de carbone dans les sols, dans les couches holorganiques et dans la végétation herbacée ont été estimés sur la base de la moyenne. Pour les boisements, les stocks de carbone dans les parties aériennes ont été assimilés à la moyenne des stocks des quatre peuplements étudiés (156,1 t/ha); le stock associé aux racines a été estimé à $21 \%$ du stock aérien (Jenkins et al., 2003) ; enfin, la quantité de carbone récoltée dans le cadre des éclaircies a été estimée en considérant les volumes éclaircis dans le cadre d'une sylviculture traditionnelle en classe de fertilité B (Colmant, 2012), une masse volumique moyenne de $420 \mathrm{~kg} / \mathrm{m}^{3}$ et une teneur en C de 52,2 \% (Ysebaert, 2012) pour un total de 47,6 t/ha.

Par rapport aux formations prairiales initiales, le reboisement en Pinus patula tend (effet juste sous le seuil de signification de $5 \%$ ) à réduire le stock de carbone du sol (ensemble des couches holorganiques et des 100 premiers centimètres). Cette tendance est liée à la diminution significative des stocks de carbone dans les 40 premiers centimètres du sol minéral. Par ailleurs, la comparaison entre les peuplements de 17 et de 25 ans suggère une réaugmentation ultérieure du stock de carbone. La contribution du sys-

\begin{abstract}
Tableau II.
Équations allométriques retenues pour les différents compartiments.

Toutes les équations sont de la forme $Y=b \times\left(C 130^{2} \times H t o t\right)$.
\end{abstract}

\begin{tabular}{|c|c|c|c|c|c|}
\hline Compartiment & $\boldsymbol{b} \pm$ erreur standard & AIC & $\mathrm{BIC}$ & $R^{2}$ & $S_{Y}$ \\
\hline Tronc & $13,35 \pm 0,48$ & 243,31 & 246,85 & 0,9144 & 0,3156 \\
\hline Branches & $4,66 \pm 0,41$ & 235,48 & 239,01 & 0,5878 & 0,5184 \\
\hline Aiguilles & $\begin{array}{l}1,51 \pm 0,13 \text { (17 ans) } \\
0,85 \pm 0,07 \text { (25 ans) }\end{array}$ & 147,79 & 152,50 & 0,6545 & 0,5932 \\
\hline
\end{tabular}




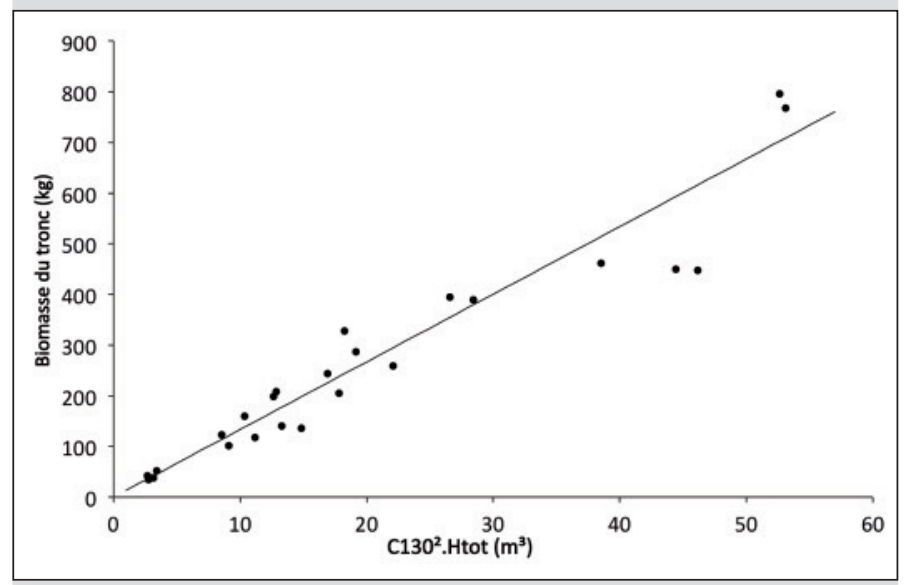

Figure 7.

Relation entre la biomasse du tronc (bois + écorce) et le prédicteur $\left(\mathrm{C}_{130^{2}} \times \mathrm{H}\right.$ tot $)$. L'équation allométrique correspondante est reprise au tableau II.

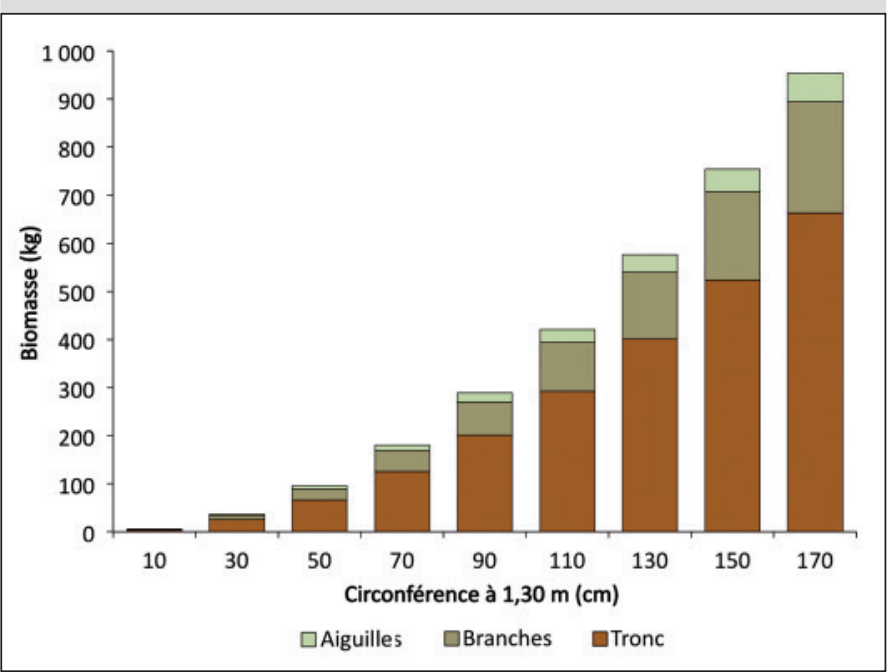

Figure 8.

Évolution de la biomasse aérienne et de sa distribution entre compartiments (tronc, branches, aiguilles) à l'échelle de l'arbre, en fonction de la circonférence à 1,3 m de hauteur dans les quatre peuplements échantillonnés.

tème racinaire $(33,0 \mathrm{t} /$ ha pour les boisements contre $5,0 \mathrm{t}$ /ha pour les pâturages) permet de réduire la différence de stock de carbone souterrain entre occupations du sol, sans toutefois parvenir à la résorber ; dans ce contexte, il est important de souligner que cette observation mériterait d'être vérifiée par des mesures de grosses racines que nous n'avons pas pu réaliser dans le cadre du présent travail.

En s'appuyant sur les facteurs influençant la production totale en volume et mis en évidence dans le cadre de l'établissement des tables de production, les stocks de carbone associés aux arbres sur pied tendent à augmenter avec l'âge des peuplements, la fertilité du site (hauteur dominante atteinte à un âge de référence donné) et la densité exprimée en termes de surface terrière. À âge et site fixés, le matériel sur pied peut donc différer sensiblement selon la densité de plantation initiale, la mortalité naturelle (avant et/ou après la fermeture du

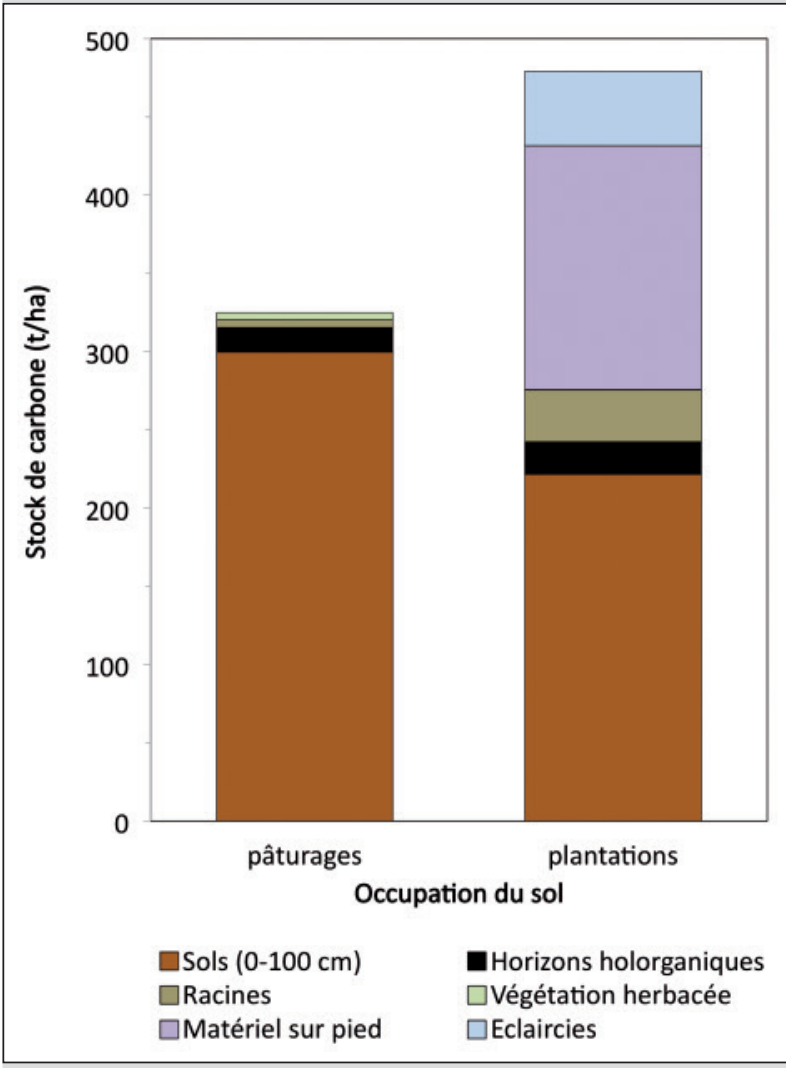

Figure 9.

Comparaison entre la capacité de séquestration de carbone des pâturages et des plantations.

couvert), et le régime d'éclaircies (fréquence, nature, intensité). Dans les peuplements étudiés, le tronc représente environ $70 \%$ du matériel sur pied, tandis que les branches et les aiguilles correspondent respectivement à $24 \%$ et $6 \%$ du total. Le tronc est envoyé en scierie et sert majoritairement à la fabrication de meubles et à la construction. Les branches sont également exportées et sont utilisées essentiellement comme bois de feu. Les aiguilles quant à elles sont laissées sur place et réapprovisionnent la couche holorganique. Le carbone contenu dans le tronc voit donc sa durée de vie prolongée à travers son stockage à plus ou moins long terme dans des meubles ou charpentes. A contrario, le carbone contenu dans les branches retourne rapidement dans l'atmosphère sous la forme de $\mathrm{CO}_{2}$ émis lors de la combustion.

En considérant une sylviculture traditionnelle et une classe de fertilité $B$, les éclaircies représenteraient environ $23 \%$ de la quantité de carbone produite dans les parties aériennes des peuplements. Selon son utilisation, la durée de vie du carbone ainsi récolté peut être plus ou moins longue, ce qui plaide pour maximiser une utilisation sous la forme de bois d'œuvre ou de produits recyclables.

Au total, la différence entre le carbone stocké dans les écosystèmes forestiers et les produits dérivés d'une part, et le carbone stocké en pâturages d'autre part, s'élève à 154 t/ha (figure 9), soit environ 7,7 t/ha/an sur la période de référence. 


\section{Références bibliographiques}

ADEFOR, 2005. Plan general de manejo forestal (PGMF) de las plantaciones de la Cooperativa agraria Jerusalén de trabajadores LTDA-Granja Porcon, establecido en convenio con el proyecto piloto forestal (PPF) y administrado por ADEFOR. Cajamarca, Pérou, Adefor.

CLUTTER J. L., 1963. Compatible growth and yield models for loblolly pine. Forest Science, 9: 354-371.

COLMANT R., 2012. Élaboration de tables de production pour le Pinus patula (Schiede ex Schltdl. \& Cham.) dans les Andes péruviennes. Mémoire de Master bioingénieur, UCL, Faculté AGRO, Belgique, $175 \mathrm{p}$.

DRIESSEN P., DECKERS J., SPAARGAREN O., NACHTERGAELE F., 2001. Lecture notes on the major soils of the world. Rome, Italie, FAO, World Soil Resources Reports 94, 334 p.

EICHHORN F., 1904. Beziehungen zwischen Bestandshöhe und Bestandsmasse. Allgemeine Forst- und Jagdzeitung, 80: 45-49.

GENET A., WERNSDÖRFER H., JONARD M., PRETZSCH H., RAUCH M., PONETTE Q., NYS C., LEGOUT A., RANGER J., VALLET P., SAINT-ANDRÉ L., 2011. Ontogeny partly explains the apparent heterogeneity of published biomass equations for Fagus sylvatica in central Europe. Forest Ecology and Management, 261: 1188-1202.

GUO L. B., GIFFORD R. M., 2002. Soil carbon stocks and land use change: a meta analysis. Global Change Biology, 8: 345-360.

GUO L. B., MENGBEN W., GIFFORD R. M., 2007. The change of soil carbon stocks and fine root dynamics after land use change from a native pasture to a pine plantation. Plant and Soil, 299: 251-262.

HEYLEN C., 2012. Impacts du reboisement (Pinus patula Schiede ex Schltdl. \& Cham.) dans les Andes péruviennes sur l'évolution des stocks de carbone dans les sols. Mémoire de Master bioingénieur, UCL, Faculté AGRO, Belgique, 114 p. + annexes.

JENKINS J. C., CHOJNACKY D. C., HEATH L. S., BIRDSEY R., 2003. National-scale biomass estimators for United States tree species. Forest Science, 49: 12-35.

JONARD M., 1999. Aménagement de peuplements de pins dans les Andes péruviennes. Mémoire de Master bioingénieur, UCL, Faculté AGRO, Belgique, 142 p.

KOMETTER R., PALOMARES B., 1994. Desarrollo Forestal Participativo en los Andes: Validación de las plantaciones comunales de la Cooperativa de trabajadores Atahualpa Jerusalén de Cajamarca.

LAGANIÈRE J., ANGERS D. A., PARÉ D., 2010. Carbon accumulation in agricultural soils after afforestation: a meta-analysis. Global Change Biology, 16: 439-453.

MINISTERIO DE AGRICULTURA, 1979. Estudio de Suelos de Granja Porcon. Lima, Pérou, Ministerio de agricultura.
RONDEUX J., 1999. La mesure des arbres et des peuplements forestiers. Gembloux, Belgique, Les Presses agronomiques de Gembloux, 544 p.

STEVENSON F. J., COLE M. A., 1999. Cycles of soil: Carbon, Nitrogen, Phosphorus, Sulfur, Micronutrients. Hoboken, NJ, USA, John Wiley \& Sons, $407 \mathrm{p}$.

SKOVSGAARD J. P., VANCLAY J. K., 2008. Forest site productivity: a review of the evolution of dendrometric concepts for evenaged stands. Forestry, 81: 13-31.

TONNEIJCK F. H., JONGMANS A. G., 2008. The influence of bioturbation on the vertical distribution of soil organic matter in volcanic ash soils: a case study in northern Ecuador. European Journal of Soil Science, 59: 1063-1075.

TURNER J., LAMBERT M., 2000. Change in organic carbon in forest plantation soils in eastern Australia. Forest Ecology and Management, 133: 231-247.

WIEDEMANN E., 1951. Ertagskundliche und waldbauliche Grundlagen der Forstwirtschaft. Francfort-sur-le-Main, Allemagne, J. D. Sauerländer's Verlag.

YSEBAERT C., 2012. Estimation des stocks de carbone dans les compartiments aériens de peuplements de Pinus patula (Schiede ex Schltdl. \& Cham.) dans les Andes péruviennes. Mémoire de Master bioingénieur, UCL, Faculté AGRO, Belgique, 115 p. + annexes. 